Article

\title{
The X-Ray Crystal Structures of Primary Aryl Substituted Selenoamides
}

\author{
Yang Li, Guo-Xiong Hua, Alexandra M. Z. Slawin and J. Derek Woollins * \\ School of Chemistry, University of St Andrews, St Andrews, Fife, Scotland, KY16 9ST, UK \\ * Author to whom correspondence should be addressed; Email: jdw3@st-andrews.ac.uk.
}

Received: 18 December 2009; in revised form: 13 February 2009 / Accepted: 19 February 2009 / Published: 23 February 2009

\begin{abstract}
The X-ray structures of 12 primary selenoamides are reported. Metric parameters are provided, together with an illustration of the range of hydrogen bonding motifs.
\end{abstract}

Keywords: Woollins reagent; Primary selenoamides; X-ray structures.

\section{Introduction}

Selenium is an essential element for life; e.g, selenocysteine is recognised as the 21 st amino acid and the importance of selenium containing enzymes in redox processes has is now recognized [1-3]. Many organoselenium compounds have been studied as biological models that simulate catalytic functions demonstrated by natural enzymes [4-14]. For example, ebselen (I, Figure 1) acts as a glutathione peroxidase $\left(\mathrm{GP}_{\mathrm{x}}\right)$ mimic and as a scavenger of peroxinitrite and the activity of the sulfur analogue of ebselen was 15 -fold lower than that of ebselen $[5,6]$. Selenazofurin (II) has been reported to be a potent inhibitor of phlebovirus infections [10]. Selenophenfurin (III) exhibits antiproliferative and inosine 5'-monophosphate dehydrogenase (IMPDH)-inhibition activity. Leukotrienes such as leukotriene $\mathrm{B}_{4}\left(\mathrm{LTB}_{4}\right)$ are important mediators of asthma, allergy, arthritis, psoriasis, and inflammatory bowel disease $[11,12]$. Galet et al. showed that benzoselenazolinones of type IV and the corresponding diselenides $\mathbf{V}$ dramatically decrease the formation of $\mathrm{LTB}_{4}$ [13]. Phenylselenosubstituted pyrimidines of type VI exhibit significant inhibitory properties on Urd Pase and TMS. Se- 
methyl selenocysteine (VII) was found to be an antitumor agent, and it has been shown that $\beta$ elimination reaction is important for this activity [14].

Figure 1. Some biologically active selenium compounds.

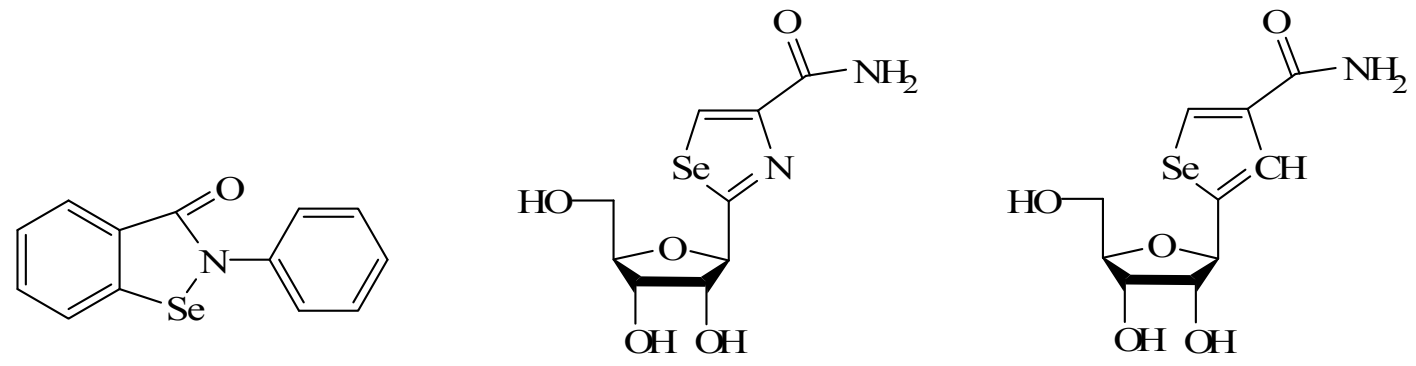<smiles>[R]C(=O)c1ccc2[nH]c(=O)sc2c1</smiles>

II

III

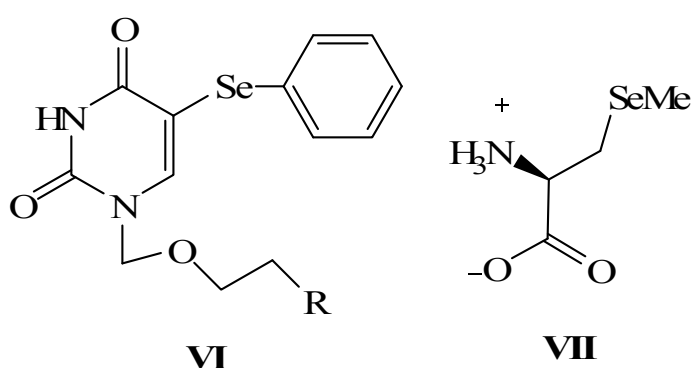

We have a long term interest in selenium chemistry [15-20]. Woollins reagent (WR, 2,4bis(phenyl)-1,3-diselenadiphosphetane-2,4-diselenide, $\left.[\mathrm{PhP}(\mathrm{Se})(\mu-\mathrm{Se})]_{2}\right)$, which is isostructural with the thionation agent $\left[\left(\mathrm{p}-\mathrm{MeOC}_{6} \mathrm{H}_{4}\right) \mathrm{P}(\mathrm{S})(\mu-\mathrm{S})\right]_{2}$ (Lawesson's reagent), and may be obtained readily from $\mathrm{PhPCl}_{2}, \mathrm{Na}_{2} \mathrm{Se}$ and $\mathrm{Se}$ [21], is an excellent selenation reagent for converting a range of unsaturated organic substrates into unusual phosphorus containing heterocycles [22-31]. We have recently reported the use of $\mathbf{W R}$ for organic transformations and for the facile synthesis of primary arylselenoamides from WR and ArCN [32]. Although the X-ray crystal structures of some tertiary and secondary selenoamides have been documented [33-39], surprisingly, no structural information has been published on primary arylselenoamides $\mathrm{ArC}(\mathrm{Se}) \mathrm{NH}_{2}$. We here provide a comparative study of a range of these systems.

\section{Results and Discussion}

Selected metric parameters for compounds $\mathbf{A}-\mathbf{L}$ are given in Table 1. The $\mathrm{C}=\mathrm{Se}$ bond lengths range from 1.822(5) to 1.856(4) $\AA$ whilst the $\mathrm{C}-\mathrm{N}$ bond lengths are in the range 1.270(7) to 1.324(8) $\AA$. This compares with literature values from the Cambridge Database for amide C-Se distances of 1.7871.885 and $\mathrm{C}-\mathrm{N}$ of $1.29-1.34 \AA$. The amide functionality is not particularly coplanar with the aryl backbone, with the selenium atom lying up to $1.406 \AA$ from the aryl ring mean plane with the C(7)$\mathrm{N}(7)-\mathrm{Se}(7)$ plane being up to $87^{\circ}$ from the aryl mean plane in this case. This maximum deviation from coplanarity may be a function of the presence of two ortho chlorine substituents in compound $\mathbf{L}$ causing repulsion. However, it is interesting to note that in compound $\mathbf{F}$ the two independent 
molecules have quite different degrees of coplanarity for the selenoamide functional group and the aryl ring suggesting that there is little electronic reason for coplanarity.

Figure 2. Examples of H-bonding motifs. (a) 1 ladder (b) 2 linked dimer (c) 7 Herringbone dimers (d) 6 tetrameric sheets (e) 10 Chains (f) 9 Helical Chains.

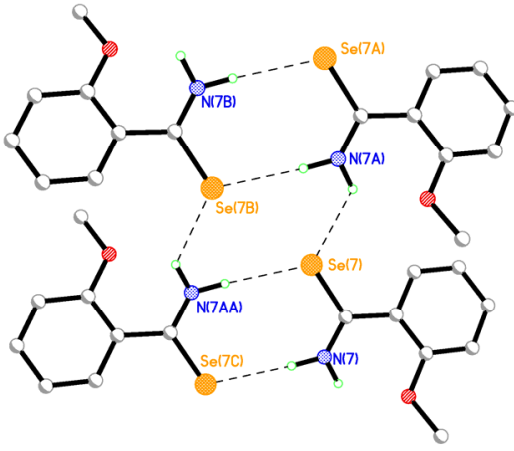

(a)

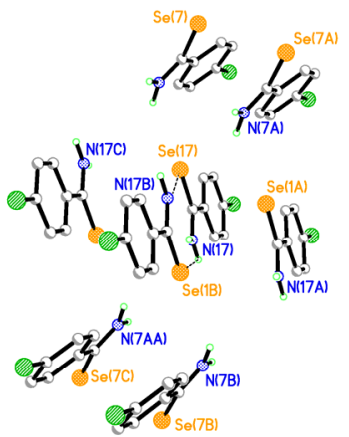

(c)

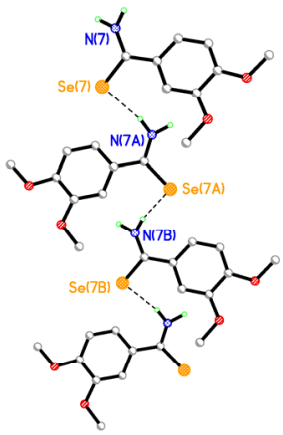

(e)

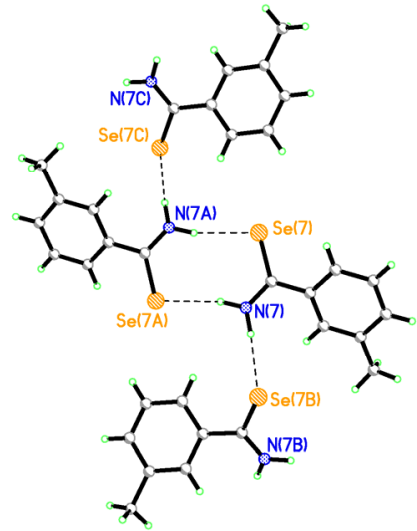

(b)

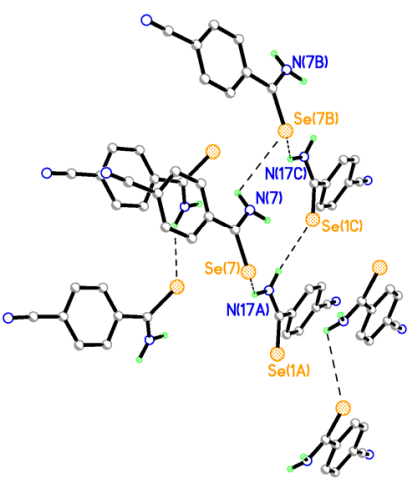

(d)

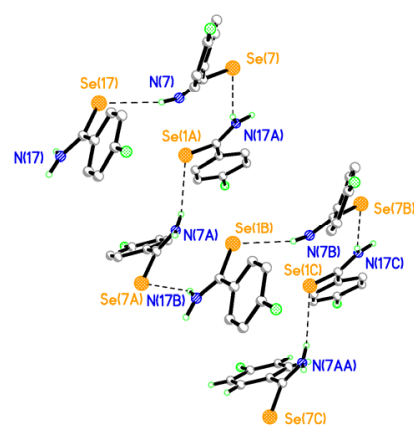

(f)

Although hydrogen bonding is well understood for $\mathrm{N}-\mathrm{H} \cdots \mathrm{O}$ and $\mathrm{N}-\mathrm{H} \cdots \mathrm{S}$ systems, there is less data available for $\mathrm{N}-\mathrm{H} \cdots$ Se systems. We have previously noted that $\mathrm{Ph}_{2} \mathrm{P}(\mathrm{Se}) \mathrm{NHP}(\mathrm{Se}) \mathrm{Ph}_{2}$ forms dimers via $\mathrm{N}-\mathrm{H} \cdots$ Se hydrogen bonds $(\mathrm{Se} \cdots \mathrm{N} 3.19, \mathrm{Se} \cdots \mathrm{H} 2.52 \AA)$ [40] and it is interesting to note the range of 
motifs that we have observed in compounds $\mathbf{A}-\mathbf{L}$ (Figure 2). We have broadly classified the pattern of hydrogen bonding in compounds $\mathbf{A}-\mathbf{L}$ and give selected parameters in Table 2 and provide illustrative examples in Figure 1. It is clear that $\mathrm{N}-\mathrm{H} \cdots$ Se hydrogen bonding is an important feature of the solid state packing of these molecules and may be a significant influence in biological systems.

Table 1. Selected ${ }^{77} \mathrm{Se}$ NMR data, bond lengths $(\AA)$ and angles $\left({ }^{\circ}\right)$ (Rows containing multiple entries are a.consequence of the presence of more than one independent molecule in the asymmetric unit).

\begin{tabular}{cccccc}
\hline & $\boldsymbol{\delta}_{\mathbf{S e}}\left(\mathbf{p p m}{ }^{*}\right)$ & $\mathbf{C}=\mathbf{S e}(\mathbf{\AA})$ & $\mathbf{C}-\mathbf{N}(\mathbf{\AA})$ & $\begin{array}{c}\text { Aryl/Scene } \\
\text { interplanar angle }\left(^{\circ}\right)\end{array}$ & $\begin{array}{c}\text { Deviation of Se from aryl } \\
\text { Mean plane }\left(^{\circ}\right)\end{array}$ \\
\hline $\mathbf{A}$ & 602.1 & $1.856(4)$ & $1.311(5)$ & 21 & 0.469 \\
$\mathbf{B}$ & 641.2 & $1.848(3)$ & $1.314(3)$ & 36 & 1.118 \\
$\mathbf{C}$ & 628.6 & $1.829(7)$ & $1.318(9)$ & 27 & 0.619 \\
$\mathbf{D}$ & 608.7 & $1.843(5)$ & $1.316(7)$ & 14 & 0.343 \\
$\mathbf{E}$ & 579.5 & $1.848(5)$ & $1.317(6)$ & 17 & 0.576 \\
$\mathbf{F}$ & 703.7 & $1.840(11)$ & $1.292(15)$ & 7 & 0.157 \\
& & $1.846(14)$ & $1.332(17)$ & 40 & 1.087 \\
$\mathbf{G}$ & 646.5 & $1.855(10)$ & $1.310(13)$ & 10 & 0.173 \\
& & $1.844(12)$ & $1.295(17)$ & 3 & 0.104 \\
$\mathbf{H} *$ & 647.2 & $1.81(5)$ & $1.27(7)$ & $8-32$ & $0.299[-0.963]$ \\
$\mathbf{I}$ & 629.6 & $1.829(6)$ & $1.324(8)$ & $37[18]$ & $1.018[0.361]$ \\
& & {$[1.838(6)]$} & {$[1.305(8)]$} & & 0.786 \\
$\mathbf{J}$ & 529.0 & $1.829(6)$ & $1.324(10)$ & 39 & 1.346 \\
$\mathbf{K}$ & 649.9 & $1.838(3)$ & $1.317(4)$ & 48 & 1.406 \\
$\mathbf{L}$ & 715.8 & $1.822(5)$ & $1.298(7)$ & 87 & \\
\hline
\end{tabular}

*Five independent molecules in the asymmetric unit, average bond lengths and ranges of $\mathrm{Se}$ deviations/interplanar angles are given.

Table 2. Major N-H $\cdots$ Se hydrogen bonding distances $(\AA)$.

\begin{tabular}{cccccccc}
\hline & Type & $\mathbf{S e} \cdots \mathbf{H}$ & $\mathbf{S e} \cdots \mathbf{N}$ & $\mathbf{S e} \cdots \mathbf{H}-\mathbf{N}$ & $\mathbf{S e} \cdots \mathbf{H}$ & $\mathbf{S e} \cdots \mathbf{N}$ & $\mathbf{S e} \cdot \mathbf{H}-\mathbf{N}$ \\
\hline $\mathbf{A}$ & Ladder & $2.55(1)$ & $3.512(3)$ & $167(3)$ & $2.72(4)$ & $3.403(3)$ & $127(3)$ \\
$\mathbf{B}$ & Linked dimers & $2.527(7)$ & $3.489(2)$ & $168(2)$ & $2.539(10)$ & $3.491(2)$ & $164(3)$ \\
$\mathbf{C}$ & Linked dimers & $2.59(3)$ & $3.510(6)$ & $156(6)$ & $2.58(3)$ & $3.491(5)$ & $155(5)$ \\
$\mathbf{D}$ & Linked dimers & $2.55(1)$ & $3.517(4)$ & $170(5)$ & $2.71(5)$ & $3.408(4)$ & $129(4)$ \\
& (sheets) & & & & & \\
$\mathbf{E}$ & Linked dimers & $2.55(7)$ & $3.527(4)$ & $174(4)$ & $2.82(5)$ & $3.415(4)$ & $120(4)$ \\
& (sheets) & & & & & \\
F & Tetramers (sheets) & $2.57(2)$ & $3.58(11)$ & $171(11)$ & $2.63(6)$ & $3.527(11)$ & $152(11)$ \\
& & $2.90(14)$ & $3.430(12)$ & $115(1)$ & $2.69(10)$ & $3.466(10)$ & $136(10)$ \\
$\mathbf{G}$ & Herringbone & $2.68(10)$ & $3.502(8)$ & $142(12)$ & $2.85(12)$ & $3.509(8)$ & $125(1)$ \\
& dimers & & & & & $160(4)$ \\
& Dimers & $2.50(17)$ & $3.43(4)$ & $158(4)$ & $2.49(16)$ & $3.43(3)$ & $169(5)$ \\
\hline
\end{tabular}


Table 2. Cont.

\begin{tabular}{|c|c|c|c|c|c|c|c|}
\hline \multirow[t]{2}{*}{$\mathbf{I}$} & Helical chain & $2.63(3)$ & $3.513(5)$ & $150(4)$ & $2.97(3)$ & $3.628(5)$ & $125(4)$ \\
\hline & & $2.74(4)$ & $3.566(5)$ & $143(5)$ & $2.62(3)$ & $3.512(5)$ & $151(5)$ \\
\hline $\mathbf{J}$ & Chain & $2.52(2)$ & $3.483(6)$ & $168(6)$ & & & \\
\hline $\mathbf{K}$ & Dimers & $2.69(1)$ & $3.63(3)$ & $162(3)$ & & & \\
\hline $\mathbf{L}$ & Linked dimers & $2.535(8)$ & $3.511(4)$ & $174(5)$ & $2.579(14)$ & $3.533(4)$ & $165(4)$ \\
\hline
\end{tabular}

\section{Experimental}

Primary arylselenoamides $\mathbf{A}-\mathbf{L}$ (Figure 3) were prepared as described previously [32]. Their X-ray crystal data (Table 3) were collected at $93 \mathrm{~K}$ by using a Rigaku MM007 High brilliance RA generator/confocal optics and Mercury CCD system. Intensities were corrected for Lorentzpolarisation and for absorption. The structures were solved by direct methods. Hydrogen atoms bound to carbon were idealised. Structural refinements were obtained with full-matrix least-squares based on $F^{2}$ by using the program SHELXTL [41]. CCDC 611494 \& 611495 CCDC 713559 - 713568 contain the supplementary crystallographic data for this paper. These data can be obtained free of charge via www.ccdc.cam.ac.uk/conts/retrieving.html or from the Cambridge Crystallographic Data centre, 12 Union Road, Cambridge CB2 1EZ, UK; fax (+44) 1223-336-033; E-mail: deposit@ccdc.cam.ac.uk.

Figure 3. The chemical structures of primary arylselenoamides $\mathbf{A}-\mathbf{L}$.

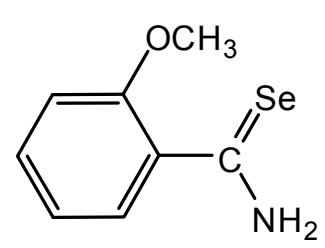

$\mathbf{A}$<smiles>COc1ccc(C(N)=[Se])cc1</smiles>

$\mathbf{E}$<smiles>NC(=[Se])c1ccc(F)cc1</smiles>

I

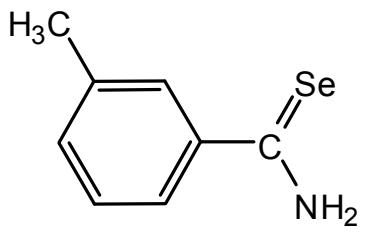

B<smiles>N#Cc1ccc(C(N)=[Se])cc1</smiles>

F<smiles>COc1ccc(C(N)=[Se])cc1OC</smiles>

J

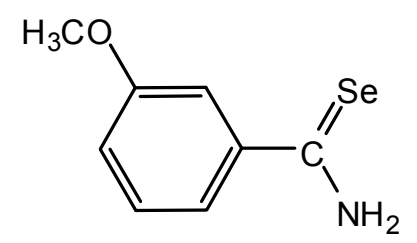

C<smiles>NC(=[Se])c1ccc(Cl)cc1</smiles>

G<smiles>COc1cccc(C(N)=[Se])c1OC</smiles>

K<smiles>Cc1ccc(C(N)=[Se])cc1</smiles>

D

$\mathrm{H}$<smiles>NC(=[Se])c1ccc(Cl)cc1Cl</smiles>

$\mathbf{L}$ 
Table 3. Details of data collections and refinements for A - $\mathbf{L}$.

\begin{tabular}{|c|c|c|c|c|c|c|}
\hline Compound & $\mathbf{A}$ & B & $\mathrm{C}$ & D & $\mathbf{E}$ & $\mathbf{F}$ \\
\hline Empirical formula & $\mathrm{C}_{8} \mathrm{H}_{9} \mathrm{NOSe}$ & $\mathrm{C}_{8} \mathrm{H}_{9} \mathrm{NSe}$ & $\mathrm{C}_{8} \mathrm{H}_{9} \mathrm{NOSe}$ & $\mathrm{C}_{8} \mathrm{H}_{9} \mathrm{NSe}$ & $\mathrm{C}_{8} \mathrm{H}_{9} \mathrm{NOSe}$ & $\mathrm{C}_{8} \mathrm{H}_{6} \mathrm{~N}_{2} \mathrm{Se}$ \\
\hline Crystal color, habit & Yellow, prism & Orange, needle & Orange, prism & Orange, needle & Yellow, platelet & Orange, needle \\
\hline Crystal dimensions/mm & $\begin{array}{l}0.20 \times 0.15 \times \\
0.05\end{array}$ & $\begin{array}{l}0.15 \times 0.15 \times \\
0.08\end{array}$ & $\begin{array}{l}0.20 \times 0.20 \times \\
0.10\end{array}$ & $\begin{array}{l}0.25 \times 0.05 \times \\
0.01\end{array}$ & $\begin{array}{l}0.20 \times 0.05 \times \\
0.02\end{array}$ & $\begin{array}{l}0.30 \times 0.06 \times \\
0.03\end{array}$ \\
\hline Crystal system & Monoclinic & Monoclinic & Orthorhombic & Monoclinic & Monoclinic & Orthorhombic \\
\hline Space group & $\mathrm{P} 2(1) / \mathrm{c}$ & $\mathrm{P} 21(1) / \mathrm{c}$ & Pbca & $\mathrm{P} 21(1) / \mathrm{c}$ & $\mathrm{P} 2(1) / \mathrm{c}$ & $\mathrm{P} 2(1) 2(1) 2(1)$ \\
\hline$a^{\prime}$ & $5.9600(11)$ & $7.5986(15)$ & $8.4086(17)$ & $9.869(2)$ & $10.108(4)$ & $7.4345(15)$ \\
\hline$b$ & $9.9600(18)$ & $10.464(2)$ & $11.586(2)$ & $6.0039(13)$ & $6.016(2)$ & $6.0647(12)$ \\
\hline$c$ & $14.114(3)$ & $10.163(2)$ & $16.960(4)$ & $13.658(3)$ & $13.765(6)$ & $34.203(6)$ \\
\hline$\beta$ & $95.506(5)$ & $96.303(6)$ & & $105.485(6)$ & $106.907(13)$ & \\
\hline$U / \AA^{3}$ & $833.9(3)$ & $803.2(3)$ & $1652.3(6)$ & $779.9(3)$ & $800.9(6)$ & $1542.1(5)$ \\
\hline$Z$ & 4 & 4 & 8 & 4 & 4 & 8 \\
\hline$M$ & 214.1 & 198.1 & 214.1 & 198.1 & 214.1 & 209.1 \\
\hline$D c / \mathrm{g} \mathrm{cm}^{-3}$ & 1.705 & 1.638 & 1.722 & 1.687 & 1.776 & 1.801 \\
\hline$\mu / \mathrm{mm}^{-1}$ & 4.441 & 4.595 & 4.483 & 4.732 & 4.625 & 4.796 \\
\hline$F(000)$ & 424 & 392 & 848 & 392 & 424 & 816 \\
\hline Measured reflections & 4447 & 4308 & 6679 & 2899 & 4170 & 8341 \\
\hline Independent reflections $\left(\mathrm{R}_{\text {int }}\right)$ & $1498(0.0393)$ & $1502(0.0396)$ & $1377(0.0908)$ & $1057(0.0393)$ & $1385(0.0629)$ & $2715(0.0562)$ \\
\hline Final R1, wR2[I>2 $\sigma(I)]$ & $0.0316,0.0971$ & $0.0269,0.0680$ & $0.0604,0.1252$ & $0.0371,0.0924$ & $0.0458,0.0911$ & $0.0752,0.1501$ \\
\hline Compound & $\mathbf{G}$ & $\mathbf{H}$ & I & $\mathbf{J}$ & $\mathbf{K}$ & $\mathbf{L}$ \\
\hline Empirical formula & $\mathrm{C}_{7} \mathrm{H}_{6} \mathrm{ClNSe}$ & $\mathrm{C}_{7} \mathrm{H}_{6} \mathrm{BrNSe}$ & $\mathrm{C}_{7} \mathrm{H}_{6} \mathrm{FNSe}$ & $\mathrm{C}_{9} \mathrm{H}_{11} \mathrm{NO}_{2} \mathrm{Se}$ & $\mathrm{C}_{9} \mathrm{H}_{11} \mathrm{NO}_{2} \mathrm{Se}$ & $\mathrm{C}_{9} \mathrm{H}_{11} \mathrm{NO}_{2} \mathrm{Se}$ \\
\hline Crystal color, habit & Orange, prism & Orange, prism & Yellow, prism & Yellow, patelet & Yellow, prism & Yellow, patelet \\
\hline Crustal dimentions $/ \mathrm{mm}$ & $0.30 \times 0.20 \times$ & $0.10 \times 0.08 \times$ & $0.30 \times 0.20 \times$ & $0.20 \times 0.20 \times$ & $0.20 \times 0.20 \times$ & $0.20 \times 0.20 \times$ \\
\hline Crystal dimentions/mm & 0.05 & 0.05 & 0.10 & 0.05 & 0.15 & 0.05 \\
\hline Crystal system & Triclinic & Triclinic & Monoclinic & Monoclinic & Monoclinic & Monoclinic \\
\hline Space group & P-1 & P-1 & $\mathrm{C} 2 / \mathrm{c}$ & $\mathrm{Cc}$ & $\mathrm{P} 2(1) / \mathrm{n}$ & $\mathrm{Cc}$ \\
\hline $\mathrm{a}$ & $4.0219(8)$ & $12.532(3)$ & $31.874(4)$ & $9.905(2)$ & $10.7387(17)$ & $9.905(2)$ \\
\hline $\mathrm{b}$ & $10.774(2)$ & $12.720(3)$ & $3.9871(6)$ & $14.311(3)$ & $7.0242(11)$ & $14.311(3)$ \\
\hline c & $17.939(4)$ & $17.285(6)$ & $22.322(3)$ & $7.1924(16)$ & $13.539(2)$ & $7.1924(16)$ \\
\hline$\alpha$ & $92.421(6)$ & $101.69(2)$ & 90 & 90 & 90 & 90 \\
\hline$\beta$ & $92.548(6)$ & $99.83(2)$ & 97.724 & $104.360(6)$ & $106.225(4)$ & $104.360(6)$ \\
\hline$\gamma$ & $91.464(6)$ & $113.572(17)$ & 90 & 90 & 90 & 90 \\
\hline $\mathrm{U} / \AA^{3}$ & $775.6(3)$ & $2373.8(11)$ & $2811.1(7)$ & $987.7(4)$ & $980.6(3)$ & $987.7(4)$ \\
\hline Z & 4 & 12 & 16 & 4 & 4 & 4 \\
\hline M & 218.5 & 263.00 & 202.1 & 244.1 & 244.1 & 244.1 \\
\hline $\mathrm{Dc} / \mathrm{g} \mathrm{cm}^{-3}$ & 1.872 & 2.208 & 1.910 & 1.642 & 1.654 & 1.642 \\
\hline$\mu / \mathrm{mm}^{-1}$ & 5.102 & 9.713 & 5.274 & 3.768 & 3.796 & 3.768 \\
\hline $\mathrm{F}(000)$ & 424 & 1488 & 1568 & 488 & 488 & 488 \\
\hline Measured reflections & 2011 & 4531 & 7115 & 2686 & 5199 & 2686 \\
\hline Independent reflections (Rint) & $1480(0.0348)$ & $3567(0.0412)$ & $2503(0.1074)$ & $1393(0.0717)$ & $1748(0.0449)$ & $1393(0.0717)$ \\
\hline Final R1, wR2[I $>2 \sigma(I)]$ & $0.0728,0.1853$ & $0.0744,0.1723$ & $0.0637,0.1540$ & $0.0431,0.1250$ & $0.0322,0.0819$ & $0.0431,0.1250$ \\
\hline
\end{tabular}

\section{Acknowledgements}

The authors are grateful to the University of St Andrews and the Engineering and Physical Science Research Council (EPSRC, U.K.) for financial support.

\section{References}

1. May, S.W.; Pollock, S.H. Selenium-Based Antihypertensives: Rationale and Potential. Drugs 1998, 56, 959-964.

2. Stadtman, T.C. Selenium Biochemistry: Mammalian Selenoenzymes. Ann. N. Y. Acad. Sci. 2000, 899, 399-402. 
3. Metanis, N.; Keinan, E.; Dawson, P.E. Synthetic Seleno-Glutaredoxin 3 Analogues Are Highly Reducing Oxidoreductases with Enhanced Catalytic Efficiency. J. Am. Chem. Soc. 2006, 128, 16684-16691.

4. Mugesh, G.; Du Mont, W.W.; Sies, H. Chemistry of Biologically Important Synthetic Organoselenium Compounds. Chem. Rev. 2001, 101, 2125-2180.

5. Ganther, H.E. Selenium-containing complexes of arginine and lysine. Bioorg. Med. Chem. 2001, 9, 1459-1466.

6. Sies, H.; Masumoto, H. Antioxidant Potential of Dimethyl Lithospermate Isolated from Salvia miltiorrhiza (Red Sage) Against Peroxynitrite. Adv. Pharmacol. 1997, 38, 2229-2246.

7. Detty, M.R.; Merkel, P.B.; Gibson, S.L.; Hilf, R. Chalcogenapyrylium dyes as dual-action sensitizers for photodynamic therapy. Oncology Res. 1992, 4, 367-373.

8. Bellnier, D.A.; Young, D.N.; Detty, M.R.; Camacho, S.; Oseroff, A.R. pH-Dependent Chalcogenopyrylium Dyes as Potential Sensitizers for Photodynamic Therapy: Selective Retention in Tumors by Exploiting $\mathrm{pH}$ Differences between Tumor and Normal Tissue. Photochem. Photobiol. 1999, 70, 630-636.

9. Leonard, K.A.; Hall, J.P.; Nelen, M.I.; Davies, S.R.; Gollnick, S.O.; Camacho, S.; Oseroff, A.R.; Gibson, S.L.; Hilf, R.; Detty, M.R. A Selenopyrylium Photosensitizer for Photodynamic Therapy Related in Structure to the Antitumor Agent AA1 with Potent in Vivo Activity and No Long-Term Skin Photosensitization. J. Med. Chem. 2000, 43, 4488-4498.

10. Smee, D.F.; Huffman, J.H.; Hall, L.L.; Huggins, J.W.; Sidwell, R.W. Inhibition of Phlebovirus infections in vivo by tiazofurin and selenazofurin. Antiviral Chem. Chemother. 1990, 1, 211-220.

11. Franchetti, P.; Cappellacci, L.; Grifantini, M.; Barzi, A.; Nocentini, G.; Yang, H.; O’Connor, A.; Jayaram, H.N.; Carrell, C.; Goldstein, B.M. Furanfurin and Thiophenfurin: Two Novel Tiazofurin Analogs. Synthesis, Structure, Antitumor Activity, and Interactions with Inosine Monophosphate Dehydrogenase. J. Med. Chem. 1995, 38, 3829-3837.

12. Franchetti, P.; Cappellacci, L.; Abu Sheikha, G.; Jayaram, H.N.; Gurudutt, V.V.; Sint, T.; Schneider, B.P.; Jones, W.D.; Goldstein, B.M.; Petra, G.; De Montis, A.; Loi, A.G.; La Colla, P.; Grifantini, M. Synthesis, Structure, and Antiproliferative Activity of Selenophenfurin, an Inosine 5'-Monophosphate Dehydrogenase Inhibitor Analogue of Selenazofurin. J. Med. Chem. 1997, 40, 1731-1737.

13. Galet, V.; Bernier, J.L.; Hénichart, J.P.; Lesieur, D.; Abadie, C.; Rochette, L.; Lindenbaum, A.; Chalas, J.; Renaud de la Faverie, J.F.; Pfeiffer, B.; Reinard, P. Benzoselenazolinone Derivatives Designed To Be Glutathione Peroxidase Mimetics Feature Inhibition of Cyclooxygenase/5Lipoxygenase Pathways and Anti-inflammatory Activity. J. Med. Chem. 1994, 37, 2903-2911.

14. Ip, C.; Hayes, C.; Budnick, R.M.; Ganther, H.E. Chemical Form of Selenium, Critical Metabolites, and Cancer Prevention. Cancer Res. 1991, 51, 595-600.

15. Fitzmaurice, J.C.; Williams, D.J.; Wood, P.T.; Woollins, J.D. Organo-phosphorus-selenium heterocycles. J. Chem. Soc., Chem. Commun. 1988, 741-743.

16. Wood, P.T.; Woollins, J.D. J. Chem. Soc., Phosphorus-selenium heterocycles. Chem. Commun. 1988, 1190-1191. 
17. Wood, P.T.; Woollins, J.D. New Phosphorus-Selenium Heterocycles. Phosphorus Sulfur Relat. Elem. 1989, 41, 51-56.

18. Pilkington, M.J.; Slawin, A.M.Z.; Williams, D.J.; Wood, P.T.; Woollins, J.D. The preparation and characterization of binary phosphorus-selenium rings. Heteroatom Chem. 1990, 1, 351-355.

19. Hall, S.W.; Pilkington, M.J.; Slawin, A.M.Z.; Williams, D.J.; Woollins, J.D. The preparation and X-ray crystal structure of $(\mathrm{PMe})_{4} \mathrm{Se}_{3}$. Polyhedron 1991, 10, 261-265.

20. Pilkington, M.J.; Slawin, A.M.Z.; Williams, D.J.; Woollins, J.D. Phosphorus-selenium heterocycles. Phosphorus, Sulfur Silicon Relat. Elem. 1992, 65, 111.

21. Gray, I.P.; Bhattacharyya, P.; Slawin, A.M.Z.; Woollins, J.D. A New Synthesis of $\left(\mathrm{PhPSe}_{2}\right)_{2}$ (Woollins Reagent) and Its Use in the Synthesis of Novel P-Se Heterocycles. Chem. Eur. J. 2005, 11, 6221-6227.

22. Bhattacharyya, P.; Woollins, J.D. Selenocarbonyl synthesis using Woollins reagent. Tetrahedron Lett. 2001, 42, 5949-5951.

23. Bhattacharyya, P.; Slawin, A.M.Z.; Woollins, J.D. The X-ray crystal structure of 2-tertbutylindolizine-3-selenoaldehyde. Inorg. Chem. Commun. 2004, 7, 1171-1173.

24. Bhattacharyya, P.; Slawin, A.M.Z.; Woollins, J.D. The Reactivity of $[\mathrm{PhP}(\mathrm{Se})(\mu-\mathrm{Se})]_{2}$ and $(\mathrm{PhP})_{3} \mathrm{Se}_{2}$ Towards Acetylenes and Cyanamides: X-ray Crystal Structures of Some P-Se-C and P-Se-C-N Heterocycles. Chem. Eur. J. 2002, 8, 2705-2711.

25. Bhattacharyya, P; Slawin, A.M.Z.; Woollins, J.D. Reaction of $\left[\left\{\mathrm{PhP}(\mathrm{Se})\left(\mathrm{L}_{-} \mathrm{Se}\right)\right\}_{2}\right]$ with Dialkyl Cyanamides: X-ray Crystal Structures of the Phosphorus-Containing Triselenapentalenes $\left[\mathrm{Me}_{2} \mathrm{~N}-\mathrm{C}(\mathrm{Se})=\mathrm{N}\right]_{2} \mathrm{P}(\mathrm{Se}) \mathrm{Ph}$ and $\left[\mathrm{O}\left(\mathrm{CH}_{2} \mathrm{CH}_{2}\right)_{2} \mathrm{~N}-\mathrm{C}(\mathrm{Se})=\mathrm{N}\right]_{2} \mathrm{P}(\mathrm{Se}) \mathrm{Ph}$. Angew. Chem., Int. Ed. 2000, 39, 1973-1975.

26. Bhattacharyya, P.; Slawin, A.M.Z.; Woollins, J.D. Bridge cleavage of $\left[\{\mathrm{PhP}(\mathrm{Se})(\mu-\mathrm{Se})\}_{2}\right]$ by $1,2-\mathrm{C}_{6} \mathrm{H}_{4}(\mathrm{EH})\left(\mathrm{E}^{\prime} \mathrm{H}\right)\left(\mathrm{E}, \mathrm{E}^{\prime}=\mathrm{O}\right.$ or $\left.\mathrm{NH}\right)$. X-ray crystal structure of $\mathrm{PhP}(\mathrm{Se})\left(\mathrm{NHC}_{6} \mathrm{H}_{4} \mathrm{NH}-1,2\right) . J$. Organomet. Chem. 2001, 623, 116-119.

27. Gray, I.P.; Slawin, A.M.Z.; Woollins, J.D. Synthesis and structure of [Fc(RO)PS $]_{2}^{-}$complexes. Dalton Trans. 2003, 3450-3457.

28. Gray, I.P.; Slawin, A.M.Z.; Woollins, J.D. Synthesis and characterisation of disulfides and esters derived from their sodium organodithiophosphonate salts. New J. Chem. 2004, 1383-1389.

29. Gray, I.P.; Slawin, A.M.Z.; Woollins, J.D. Synthesis and structure of [An(RO)PS $]^{-}$complexes. Dalton Trans. 2004, 2477-2486.

30. Hua, G.; Li, Y.; Slawin, A.M.Z.; Woollins, J.D. Stereoselective synthesis of olefins by a reductive coupling reaction. Dalton Trans. 2007, 1477-1480.

31. Hua, G.; Woollins, J.D. The syntheses of sulfides by deoxygenation of sulfoxides using Woollins' reagent. Tetrahedron Lett. 2007, 48, 3677-3679.

32. Hua, G.; Li, Y.; Slawin, A.M.Z.; Woollins, J.D. Synthesis of Primary Arylselenoamides by Reaction of Aryl Nitriles with Woollins' Reagent. Org. Lett. 2006, 8, 5251-5254.

33. Otten, P.A.; Gorter, S.; Van der Gen, A. A Structural Study of Selenobenzamides: Crystal Structures and Dynamic ${ }^{13}$ C NMR. Chem. Ber. 1997, 130, 49-54.

34. Li, G.; Reibenspies, J.H.; Derecskei-Kovacs, A.; Zingaro, R.A. N,N-dimethyl-selenobenzamide: two solid phases and low-temperature phase change Polyhedron 1999, 18, 3391. 
35. Fischer, H.; Triliomis, A.; Gerbing, U.; Huber, B.; Mueller, G. Thio- and seleno-acrylamide derivatives from ynamines and transition metal-co-ordinated thio- and seleno-aldehydes and ketones. J. Chem. Soc., Chem. Commun. 1987, 559-560.

36. Fischer, H.; Gerbing, U.; Triliomis, A.; Mueller, G.; Huber, B.; Riede, J.; Hofmann, J.; Burger, P. Übergangsmetall-koordinierte Heteroolefine als Synthesebausteine: Thio- und Selenoacrylamide aus 1-(Diethylamino)-1-propin und Heteroaldehyd- und -keton-Komplexen. Chem. Ber. 1988, 121, 2095.

37. Murai, T.; Mizutani, T.; Kanda, T.; Kato, S. Reactions of diselenoic acid esters with amines and X-ray crystal structure analyses of aromatic selenoamides. Heteroatam Chem. 1995, 6, 241-246.

38. Blau, H.; Grobe, J.; Le Van, D.; Krebs, B.; Lage, M. Reactive E=C [p-p] $\pi$ Systems, 46 Syntheses and X-ray Crystal-Structure Analyses of Trifluoromethyl-Substituted Ketiminodiselanes, Selenoamides and Selenoacrylamides. Chem. Ber. 1997, 130, 913-922;

39. Murai, T.; Niwa, N.; Ezaka, T.; Kato, S. Structure of N-Aryl Selenoacetamides in Solutions and in the Solid State. J. Org. Chem. 1998, 63, 374-376.

40. Bhattacharyya, P.; Novosad, J.; Phillips, J.; Slawin, A.M.Z.; Williams, D. J.; Woollins, J. D. Bis(bidentate) complexes of iminobis(diphenylphosphine chalcogenides) $\left[\mathrm{M}\left\{\mathrm{N}\left(\mathrm{XPPh}_{2}\right)_{2}-X, X^{\prime}\right.\right.$ \}$\left._{2}\right](\mathrm{X}=\mathrm{S}$ or Se; M = Ni, Pd or Pt). J. Chem. Soc., Dalton Trans. 1995, 1607-1613.

41. SHELXTL 6.11. Bruker AXS: Madison, WI, USA, 2004.

Sample Availability: Available from the authors.

(C) 2009 by the authors; licensee Molecular Diversity Preservation International, Basel, Switzerland. This article is an open-access article distributed under the terms and conditions of the Creative Commons Attribution license (http://creativecommons.org/licenses/by/3.0/). 